\title{
Amino-acid Pool Composition of Saccharomyces cerevisiae as a Function of Growth Rate and Amino-acid Nitrogen Source
}

\author{
By T. G. WATSON \\ National Food Research Institute, Council for Scientific and Industrial \\ Research, P.O. Box 395, Pretoria 000 I, Republic of South Africa
}

(Received 23 March 1976; revised II May 1976)

\begin{abstract}
SUMMARY
The composition of the amino-acid pool of Saccharomyces cerevisiae is markedly influenced by the amino-acid nitrogen source. The yeast tends to accumulate the amino acid supplied and those closely related to it metabolically. A relatively high concentration of glutamic acid is maintained in the pools of all cultures irrespective of the nitrogen source, reflecting the central role of glutamic acid in nitrogen metabolism. The total amino-acid pool concentration increases as a function of growth rate, although differences exist in the behaviour of individual amino acids.
\end{abstract}

\section{INTRODUCTION}

Recent studies on the accumulation of amino acids by growing yeasts have concentrated mainly on the use of $\mathrm{NH}_{4}^{+}$as sole nitrogen source to avoid problems arising from permeation and transport phenomena. Dawson (1965) showed that the amino-acid pool of Candida utilis varied with growth rate and carbon source. In an $\mathrm{NH}_{4}^{+}$-limited continuous culture with glucose as the carbon source, alanine and glutamic acid were predominant and increased with growth rate whereas aspartic acid decreased. Valine, threonine, proline and histidine were prominent; as also were glycine, serine and leucine at the faster growth rates, and phenylalanine at the slower growth rates. Moat et al. (1969) examined the pool amino acids of Saccharomyces cerevisiae grown in batch culture with $\mathrm{NH}_{4}^{+}$as the sole nitrogen source for $\mathrm{I} 8$ to $\mathrm{I} 4 \mathrm{O} \mathrm{h}$. Alanine, arginine and glutamate were found in relatively high concentrations; the other amino acids were found in smaller, although significant, amounts. The aminoacid concentration was usually highest at the earliest time of harvest and gradually declined after prolonged incubation. Alanine remained at a high concentration throughout and the aspartate concentration, which was relatively low at $18 \mathrm{~h}$, increased to a peak at $43 \mathrm{~h}$ and then declined.

Where individual amino acids have been used as the sole nitrogen source, a comparative study has not been the purpose of the investigation. For example, Nurse \& Wiemken (1974) have examined the changes occurring in the amino-acid pool of arginine-grown Candida utilis during the cell-division cycle, and Brown, Satyanarayana \& Umbarger (1975) included amino-acid pool analyses in their study of the biosynthesis of leucine biosynthetic enzymes during growth of Saccharomyces cerevisiae on leucine.

This communication relates to the effect of various individual amino-acid nitrogen sources on the intracellular amino-acid pool levels of Saccharomyces cerevisiae during early-exponential growth in batch culture; and to the accumulation of individual amino acids as a function of the steady-state exponential growth rate in a nitrogen-limited continuous culture, using glutamate as the sole nitrogen source. 


\section{METHODS}

Organism. The organism used was Saccharomyces cerevisiae 448 from the culture collection of the Microbiology Research Group of the South African Council for Scientific and Industrial Research, Pretoria.

Growth conditions. The growth medium consisted of : 4 mM- $\mathrm{MgSO}_{4} \cdot 7 \mathrm{H}_{2} \mathrm{O} ; 20 \mathrm{~mm}-\mathrm{KH}_{2} \mathrm{PO}_{4}$; I mM- $\mathrm{CaCl}_{2} \cdot 2 \mathrm{H}_{2} \mathrm{O}$; stock trace element solution (I $\left.\mathrm{ml} \mathrm{l}^{-1}\right)$; lactic acid $\left(3 \mathrm{ml} \mathrm{l}^{-1}\right)$; and sodium lactate to $\mathrm{pH} 4.0$. It was sterilized by autoclaving; and then filter-sterilized stock vitamin solution ( $\left.\mathrm{I} \mathrm{ml} \mathrm{l}^{-1}\right)$, glucose ( $\left.\mathrm{I} \% \mathrm{w} / \mathrm{v}\right)$ and amino-acid nitrogen source ( $2 \mathrm{mM}$ ) were added. The stock vitamin solution contained $(\mathrm{mg} / 250 \mathrm{ml}$ water): biotin, 5 ; calcium pantothenate, 500; folic acid, 5; inositol, 2500; nicotinic acid, 100; 4-aminobenzoic acid, 50; pyridoxin- $\mathrm{HCl}$, I00; riboflavin, 50 ; thiamin $-\mathrm{HCl}$, 100. The stock trace element solution contained (mg/250 ml water): boric acid, I25; cupric sulphate, I0; potassium iodide, 25; ferric chloride, 50; manganese sulphate, I00; sodium molybdate, 50; zinc sulphate, I00.

Continuous cultures were grown in a New Brunswick Magnaferm fermenter (New Brunswick Scientific Co., New Jersey, U.S.A.) operated as a chemostat at $30^{\circ} \mathrm{C}$, with a working volume of $\mathrm{I} \cdot 31$ aerated at I $1 \mathrm{~min}^{-1}$ and stirred at $400 \mathrm{rev} . \mathrm{min}^{-1}$. At least 3 days were allowed for the cultures to stabilize after a shift in dilution rate before samples were taken. Replicate determinations at a given dilution rate were made at intervals of not less than one day. Transient states between shifts in dilution rate were not examined.

Batch cultures ( $100 \mathrm{ml}$ ) were grown in $250 \mathrm{ml}$ Erlenmeyer flasks with shaking at $30^{\circ} \mathrm{C}$. Yeast was inoculated at a low concentration to give approximately $0.15 \mathrm{mg}$ dry $\mathrm{wt} \mathrm{ml}^{-1}$ after $\mathrm{I} 6 \mathrm{~h}$ when the organisms were harvested. Dry weight was determined by filtering samples through pre-weighed Millipore filters ( $\mathrm{I} \cdot 2 \mu \mathrm{m}$ pore size), washing the organisms with 2 vols distilled water, and drying to constant weight at $80^{\circ} \mathrm{C}$.

In the starvation experiment, yeast was grown batchwise in basal medium containing 2 mM-glutamate as the sole nitrogen source, and harvested by membrane filtration, in lots of I00 $\mathrm{ml}$, when the concentration of organisms reached $0.15 \mathrm{mg}$ dry wt $\mathrm{ml}^{-1}$. The yeast was then washed from the filters into $100 \mathrm{ml}$ nitrogen-free basal medium in a $250 \mathrm{ml}$ Erlenmeyer flask, and shaken at $30^{\circ} \mathrm{C}$. Samples were taken for amino-acid analysis at the times indicated.

Determination of amino-acid pools. A known volume of culture, usually $50 \mathrm{ml}$, containing about $7.5 \mathrm{mg}$ dry wt yeast from the batch cultures or to to $30 \mathrm{mg}$ dry wt from the continuous cultures, depending on dilution rate, was filtered through a $47 \mathrm{~mm}$ Millipore filter $(\mathrm{I} \cdot 2 \mu \mathrm{m}$ pore size) and washed twice with $100 \mathrm{ml}$ ice-cold water. The complete operation took between 15 and $30 \mathrm{~s}$. No amino acids were lost during the washing procedure. The filter was then boiled for $15 \mathrm{~min}$ in approximately $8 \mathrm{ml}$ water. The yeast was then washed from the filter with further water, and the combined filtrates were centrifuged and made up to ro $\mathrm{ml}$. Amino acids were analysed using a Technicon amino-acid analyser. Amino-acid concentrations of less than $0.05 \mu \mathrm{mol} / 100 \mathrm{mg}$ dry wt were not recorded.

Growth rates. The specific growth rate $(\mu)$ of batch cultures was determined spectrophotometrically during early-exponential growth when the yeast concentration was less than $0 \cdot 1 \mathrm{mg} \mathrm{ml}{ }^{-1}$, using the relationship:

$$
\mu=\left(\ln x_{2}-\ln x_{1}\right) /\left(t_{2}-t_{1}\right)
$$

in which $x_{2}$ and $x_{1}$ are the absorbances at $625 \mathrm{~nm}$ at times $t_{2}$ and $t_{1}$ respectively. In a chemostat culture, $\mu=D$, the dilution rate, under steady-state conditions (Herbert, 1958). 


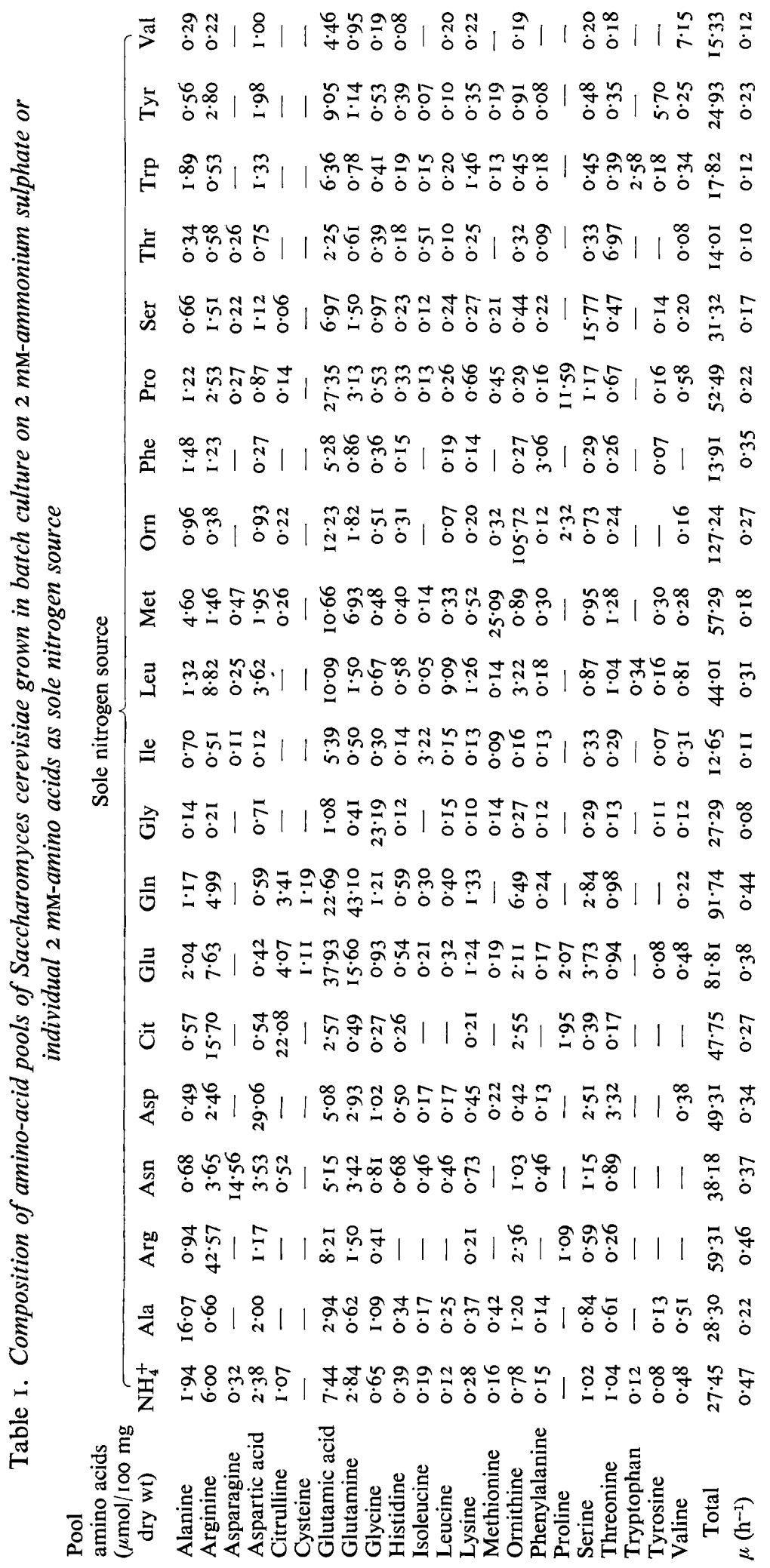




\section{RESULTS}

\section{Effect of individual amino-acid nitrogen sources (Table I)}

Saccharomyces cerevisiae was grown in batch culture on ammonium sulphate and on each of 19 amino acids as sole nitrogen source. The maximum specific growth rates ranged between $0.08 \mathrm{~h}^{-1}$ and $0.47 \mathrm{~h}^{-1}$, being lowest for glycine and highest for ammonium sulphate and arginine. Growth did not occur on cysteine, histidine or lysine.

The major amino acid in any particular pool was usually that used as the nitrogen source. Exceptions were isoleucine, leucine, phenylalanine, proline, tryptophan and tyrosine. In these cases, glutamic acid was the major amino acid and the acids used as the nitrogen source had the second highest concentration in the particular pool. Each amino acid was found in its highest concentration in the pool of organisms fed with that amino acid. The yeast also tended to accumulate amino acids closely related metabolically to the nitrogen source. For example, proline accumulated in the pools of cultures grown on arginine, citrulline, glutamic acid, ornithine or proline, but was not detected in the others. Similarly, cultures grown on aspartic acid accumulated threonine in addition to aspartic acid, and those grown on citrulline accumulated arginine as well as citrulline.

Many amino acids were detected in the pools of all cultures. Others, namely asparagine, citrulline, cysteine, histidine, isoleucine, leucine, methionine, phenylalanine, proline, tryptophan, tyrosine and valine, were not found in all pools. Irrespective of the nitrogen source, glutamic acid was always present at a relatively high concentration, being the highest or second highest in concentration, except when citrulline was the nitrogen source, it was third highest.

\section{Effect of growth rate (Table 2)}

The total concentration of amino acids in the pool of yeast grown in continuous culture on glutamate as sole nitrogen source increased approximately Io-fold between $\mu=0.04 \mathrm{~h}^{-1}$ and $\mu=0.36 \mathrm{~h}^{-1}$. Glutamic acid was the major pool amino acid throughout, and represented approximately one half of the total pool. Some amino acids, however, changed their relative contribution to the pool with change in growth rate. For example, aspartic acid was prominent at low growth rates and arginine, glutamine and ornithine at high growth rates. Citrulline, cysteine and proline could be detected only at higher growth rates and asparagine, methionine and tyrosine at lower growth rates.

\section{Effect of nitrogen starvation (Table 3)}

Initially, glutamic acid was rapidly metabolized, although it was not completely exhausted even after Io $h$ starvation. Later, glutamine and arginine were utilized. During the period when arginine was being depleted most rapidly, ornithine accumulated and, after io $\mathrm{h}$ starvation, became the major pool constituent.

Although the total pool concentration fell from $10.44 \mu \mathrm{mol} / \mathrm{roo} \mathrm{ml}$ culture to $4.54 \mu \mathrm{mol} /$ $100 \mathrm{ml}$ culture after starvation for $15 \mathrm{~min}$, the level of aspartic acid rose from $0.06 \mu \mathrm{mol} /$ $100 \mathrm{ml}$ to $0.14 \mu \mathrm{mol} / \mathrm{I} 00 \mathrm{ml}$. Even after starvation for $10 \mathrm{~h}$, when the total amino-acid pool level had dropped to $0.7 \mathrm{I} \mu \mathrm{mol} / 100 \mathrm{ml}$, aspartic acid, at $0.08 \mu \mathrm{mol} / \mathrm{ro0} \mathrm{ml}$, was still above its starting concentration. 
Table 2. Effect of specific growth rate on the amino-acid pool composition of Saccharomyces cerevisiae grown in a nitrogen-limited continuous culture with $2 \mathrm{mM}$-glutamate as the sole nitrogen source

Pool amino acids $(\mu \mathrm{mol} / \mathrm{I} 00 \mathrm{mg}$ dry $w t)$

Alanine

Arginine

Asparagine

Aspartic acid

Citrulline

Cysteine

Glutamic acid

Glutamine

Glycine

Histidine

Isoleucine

Leucine

Lysine

Methionine

Ornithine

Phenylalanine

Proline

Serine

Threonine

Tryptophan

Tyrosine

Valine

Total

No. of determinations

\begin{tabular}{|c|c|c|c|c|c|c|}
\hline \multicolumn{7}{|c|}{ Specific growth rate $\left(\mathrm{h}^{-1}\right)$} \\
\hline 0.04 & 0.10 & 0.20 & 0.26 & 0.29 & 0.34 & 0.36 \\
\hline 0.30 & $0.4 \mathrm{I}$ & 0.94 & $I \cdot 04$ & $2 \cdot 16$ & $I \cdot 74$ & $\mathrm{I} \cdot 94$ \\
\hline 0.07 & 0.13 & $2 \cdot 4 I$ & 3.06 & $5 \cdot 55$ & $6 \cdot 54$ & $6 \cdot 52$ \\
\hline 0.09 & 一 & - & 一 & - & - & - \\
\hline 0.59 & 0.64 & $\mathrm{I} \cdot 05$ & 0.68 & 0.35 & 0.23 & $0 \cdot 34$ \\
\hline - & - & 0.08 & 0.23 & 0.77 & $2 \cdot 06$ & $2 \cdot 12$ \\
\hline- & - & - & - & 0.43 & 0.70 & 0.94 \\
\hline $3 \cdot 59$ & $7 \cdot 31$ & $15 \cdot 91$ & I $6 \cdot 24$ & $23 \cdot 37$ & 25.09 & $27 \cdot 93$ \\
\hline $0 \cdot 16$ & 0.52 & 3.63 & $4 \cdot 28$ & $8 \cdot 79$ & $8 \cdot 67$ & $9 \cdot 97$ \\
\hline 0.10 & $0 \cdot 13$ & 0.39 & 0.46 & 0.39 & 0.56 & 0.62 \\
\hline 0.14 & 0.06 & 0.25 & 0.37 & 0.22 & 0.33 & 0.42 \\
\hline 0.07 & 0.06 & 0.11 & 0.06 & 0.10 & 0.10 & O.I I \\
\hline 0.17 & 0.12 & 0.23 & 0.25 & 0.23 & 0.21 & 0.22 \\
\hline 0.09 & $0.1 \mathrm{I}$ & 0.34 & 0.09 & 0.39 & 0.52 & 0.80 \\
\hline 0.05 & - & - & - & 0.08 & 0.14 & - \\
\hline 0.05 & 0.09 & 0.58 & 0.79 & $I \cdot I 3$ & I. 66 & $2 \cdot 12$ \\
\hline $0.1 \mathrm{I}$ & 0.08 & 0.13 & 0.17 & 0.07 & 0.07 & 0.12 \\
\hline- & - & 0.07 & 0.53 & 0.95 & $1 \cdot 13$ & $2 \cdot 2 \mathrm{I}$ \\
\hline 0.15 & 0.24 & 0.73 & 0.86 & $\mathrm{I} \cdot 88$ & $1 \cdot 80$ & 2.59 \\
\hline 0.10 & 0.15 & 0.45 & 0.41 & 0.68 & 0.43 & 0.50 \\
\hline- & - & 0.20 & 0.16 & I. 53 & 0.55 & - \\
\hline 0.06 & 0.05 & 0.09 & 0.09 & 0.07 & 0.07 & - \\
\hline 0.17 & 0.19 & 0.45 & 0.39 & 0.32 & 0.36 & 0.24 \\
\hline $6 \cdot 06$ & $10 \cdot 29$ & $28 \cdot 04$ & $30 \cdot 16$ & $49 \cdot 46$ & $52 \cdot 96$ & $59 \cdot 71$ \\
\hline 7 & 4 & 4 & I & 3 & 2 & \\
\hline
\end{tabular}

Table 3. Effect of nitrogen starvation on the amino-acid pool composition of Saccharomyces cerevisiae grown on $2 \mathrm{mM}$-glutamate as the sole nitrogen source

Pool amino acids ( $\mu \mathrm{mol} / 100 \mathrm{ml}$ culture)

Alanine

Arginine

Asparagine

Aspartic acid

Citrulline

Cysteine

Glutamic acid

Glutamine

Glycine

Histidine

Isoleucine

Leucine

Lysine

Methionine

Ornithine

Phenylalanine

Proline

Serine

Threonine

Tryptophan

Tyrosine

Valine

Total
Time of starvation (h)

\begin{tabular}{ccccccc}
\hline 0 & 0.25 & 0.50 & 1.00 & 2.50 & 5.00 & 10.00 \\
0.19 & 0.06 & 0.04 & 0.05 & 0.05 & 0.04 & 0.03 \\
1.10 & 1.00 & 0.73 & 0.18 & 0.02 & - & - \\
\hline 0.06 & - & - & - & - & - & - \\
0.47 & 0.14 & 0.14 & 0.18 & 0.11 & 0.12 & 0.08 \\
0.10 & 0.05 & 0.10 & - & - & - & - \\
4.30 & 0.63 & 0.48 & 0.48 & 0.23 & 0.24 & 0.10 \\
2.36 & 1.68 & 1.23 & 0.11 & 0.03 & 0.02 & - \\
0.13 & 0.04 & 0.04 & 0.05 & 0.03 & 0.05 & 0.04 \\
0.08 & 0.03 & - & - & - & - & - \\
0.02 & - & - & 0.02 & 0.02 & 0.04 & 0.03 \\
0.04 & 0.02 & 0.02 & - & 0.05 & 0.07 & 0.06 \\
0.18 & 0.10 & 0.02 & 0.04 & - & - & - \\
0.03 & 0.05 & 0.03 & 0.01 & 0.01 & 0.01 & 0.01 \\
0.31 & 0.18 & 0.35 & 0.04 & 0.14 & 0.16 & 0.14 \\
0.01 & 0.02 & 0.02 & 0.02 & 0.03 & 0.06 & 0.05 \\
0.43 & 0.09 & 0.09 & - & - & - & - \\
0.46 & 0.07 & 0.05 & 0.06 & 0.05 & 0.06 & 0.05 \\
0.09 & 0.03 & 0.03 & 0.04 & 0.03 & 0.03 & 0.03 \\
- & - & - & - & - & - & - \\
- & 0.01 & 0.01 & 0.01 & 0.01 & 0.03 & 0.02 \\
0.08 & 0.03 & 0.02 & 0.02 & 0.03 & 0.02 & 0.03 \\
10.44 & 4.54 & 3.42 & 1.33 & 0.88 & 1.04 & 0.71
\end{tabular}




\section{DISCUSSION}

The relatively high concentration of glutamic acid maintained in the amino-acid pools of all cultures, irrespective of the nitrogen source, reflects the central role of glutamic acid in nitrogen metabolism.

The levels of the major pool amino acids occurring in yeast grown on various individual amino-acid nitrogen sources are indicative of general metabolic inter-amino-acid conversions and not a requirement for protein biosynthesis. This latter requirement is probably met by much smaller concentrations of amino acids and may be reflected in the levels of the metabolically relatively-inactive amino acids. Wiemken \& Nurse (1973) have shown that more than one type of amino-acid pool exists in yeast and that the pools differ in function. No attempt was made in the present study to differentiate between these pools.

The increase in the total amino-acid pool as a function of growth rate was demonstrated in continuous culture using a single amino-acid nitrogen source. A similar interpretation of the batch culture results, using different individual amino-acid nitrogen sources, could not be made because of the overriding effect of the nitrogen source on pool constituents.

The increased concentration of many amino acids at higher growth rates is probably associated with increased enzyme activity.

Certain amino acids, especially histidine, isoleucine, leucine, methionine, phenylalanine, threonine, tryptophan, tyrosine and valine, did not increase, or increased less with increasing growth rate than the total amino-acid pool. These amino acids were relatively minor pool constituents, representing some of the less metabolically-active amino acids, and in many cases corresponded with those amino acids which were poorly utilized during nitrogen starvation. Other amino acids, namely arginine, citrulline, glutamine, ornithine and proline, accumulated to a relatively greater extent. The concentration of arginine, for example, increased nearly Ioo-fold. It would seem that the higher level of glutamic acid required at increased growth rates unavoidably leads to the accumulation of closely related amino acids as a result of inefficient feed back control of their synthesis.

The lower concentration of aspartic acid at higher growth rate is surprising in view of the important role of this amino acid in transamination. It is interesting to note, however, that nitrogen starvation also leads to a net synthesis of this amino acid.

I am grateful to Celia Hollingworth, Doreen Morgan and L. Pretorius for valuable technical assistance.

\section{REFERENCES}

Brown, H. D., Satyanarayana, T. \& Umbarger, H. E. (I975). Biosynthesis of branched-chain amino acids in yeast: effect of carbon source on leucine biosynthetic enzymes. Journal of Bacteriology 12r, 959-969.

DAwson, P.S. S. (1965). The intracellular amino acid pool of Candida utilis during growth in batch and continuous flow cultures. Biochimica et biophysica acta III, 51-66.

HerberT, D. (1958). Some principles of continuous culture. In Recent Progress in Microbiology, pp. $38 \mathrm{I}-396$. Edited by G. Turner. Oxford: Blackwell Scientific Publications.

Moat, A. G., Ahmad, F., Alexander, J. K. \& Barnes, J. J. (1969). Alteration in the amino acid content of yeast during growth under various nutritional conditions. Journal of Bacteriology 98, 573-578.

NuRSE, P. \& WiemKen, A. (1974). Amino acid pools and metabolism during the cell division cycle of argininegrown Candida utilis. Journal of Bacteriology 117, I $108-1$ I 16.

Wiemken, A. \& Nurse, P. (1973). Isolation and characterization of the amino acid pools located within the cytoplasm and vacuoles of Candida utilis. Planta 109, 293-306. 